\title{
Incidence of gastric cancer, its subtypes, and correlation with Helicobacter Pylori
}

\author{
KC Shiva Raj ${ }^{1}$, Amatya $\mathrm{GL}^{2}$, Lakhey A ${ }^{3}$, Basnet $\mathrm{S}^{3}$, Aryal $\mathrm{G}^{3}$ \\ ${ }^{1}$ Consultant Pathologist, GRP Polyclinic Pvt. Ltd., Kathmandu, Nepal \\ ${ }^{2}$ Consultant Gastroenterologist, GRP Polyclinic Pvt. Ltd., Kathmandu, Nepal \\ ${ }^{3}$ Consultant Pathologist, Kist Medical College and Teaching Hospital, Lalitpur, Nepal
}

\section{Keywords: \\ Cancer; \\ Lauren; \\ Hepatoid adenocarcinoma; \\ Papillary carcinoma; \\ Signet ring; \\ Stomach; \\ Tubular adenocarcinoma}

\begin{abstract}
Background: Gastric cancer is the fourth most commonly diagnosed cancer and the second most common cause of cancer related death worldwide. It is the common cause of cancer related death in Nepal. Helicobacter Pylori has been classified as a definite carcinogen along with other factors. The aim of this study was to find the incidence of gastric cancer among the patients undergoing upper gastroscopy, its various subtypes and association with Helicobacter Pylori.
\end{abstract}

Materials and Methods: This is a retrospective and prospective study carried out at GRP Polyclinic and Kist Medical College Teaching Hospital. All the patients undergoing upper gastrointestinal endoscopy were included in this study. Data of all the gastric endoscopic biopsies done from June 2011 to January 2013 were collected and analyzed. All the biopsy specimens were processed routinely in histopathology laboratory. Specimens showing carcinoma were enrolled in this study and all the relevant demographic data were collected.

Results: Out of 3395 biopsy cases; 49 cases (1.44\%) were diagnosed as adenocarcinoma stomach. The overall mean age for carcinoma was 47.6 years with a mild male preponderance. Thirty cases $(61.2 \%)$ were of intestinal type, $(n=11 ; 22.4 \%)$ were of diffuse type and $(n=8 ; 16.3 \%)$ were mixed type of adenocarcinoma. According to WHO classification the most common subtype was tubular adenocarcinoma $(n=35 ; 71.5 \%)$ followed by signet ring type (11 cases; $22.4 \%)$. Out of 49 cases of adenocarcinoma stomach 39 cases (79.5\%) were Helicobacter Pylori positive.

Conclusion: This study shows that gastric carcinoma is a male predominant neoplasm usually of old age but can occur at younger ages. It predominantly occurs in Helicobacter Pylori infected patients and $\mathrm{H}$. Pylori eradication will help to decrease the incidence rate and mortality of stomach cancer.

\section{INTRODUCTION}

Gastric cancer is the $4^{\text {th }}$ most commonly diagnosed cancer and the $2^{\text {nd }}$ most common cause of cancer related death worldwide. ${ }^{1,2}$ Studies done in Nepal also showed carcinoma stomach as the $3^{\text {rd }}$ most common cancer in males preceded by lung cancer and oral cancers where as it is the $2^{\text {nd }}$ most

\section{Correspondence:}

Dr. Shiva Raj KC, $M D$

Department of Pathology. Kist Medical College and Teaching Hospital, Lalitpur, Nepal.

Email: shiva_kc_123@yahoo.com common cause of cancer related mortality in males.In females, it is the $6^{\text {th }}$ most common cancer and is the $5^{\text {th }}$ most common cause of mortality. ${ }^{2,3}$

Carcinoma stomach is the third most common cause of death worldwide after carcinoma colon and prostate cancer. ${ }^{3}$ The burden of cancer is increasing in economically developing countries as a result of population aging and growth as well as, change in lifestyle including food habits, smoking, physical inactivity, and "westernized" diets. ${ }^{4}$ 
Gastric carcinogenesis is a multistep multifactorial disease. In 1994, WHO and IARC consensus group 5 classified Helicobacter pylori (H. Pylori) as a definite carcinogen. ${ }^{5}$ Since then several studies done worldwide has shown that H. Pylori was associated with gastric cancer. ${ }^{6-8}$ However, in some studies there is a striking contrast between the total number of persons infected with $\mathrm{H}$. pylori and those subsequently developing gastric cancer. Only 1 out of $1000 \mathrm{H}$. Pylori case has found to develop gastric cancer in Germany and even less in the United States which indicates the development of gastric cancer is multi-factorial process out of which H. Pylori is one factor. ${ }^{9}$

The 2010 WHO classification classifies gastric cancers into four major histologic patterns: tubular, papillary, mucinous and poorly cohesive (including signet ring cell carcinoma), along with uncommon histological variants. ${ }^{10}$ Similarly Lauren classifies gastric cancer into intestinal type and diffuse type. Intestinal cancer is often related to environmental factors such as $\mathrm{H}$. pylori infection, diet, and life style, the diffuse type is more often associated with genetic abnormalities. ${ }^{10}$

\section{MATERIALS AND METHODS}

This was a retrospective and prospective study carried out at GRP Polyclinic and Kist Medical College Teaching Hospital. Data of all the gastric endoscopic biopsies done from June 2011 to January 2013 were collected and analyzed. All the biopsy specimens were processed routinely in histopathology laboratory. One slide of Hematoxylin and Eosin (HE) and one slide of Giemsa stained slide were prepared along with Periodic stain with Schiff's stain (PAS) were used wherever necessary. The slides were reviewed by consultant pathologists. Specimens diagnosed as adenocarcinoma were enrolled in this study and all the relevant clerical data were collected. The data were analyzed using SPSS version 17.0.

\section{RESULTS}

During the period 3395 endoscopic biopsies were reviewed out of which 49 cases $(1.44 \%)$ were of adenocarcinoma stomach. The overall mean age for carcinoma was 47.6 years with 15 years being the youngest and 77 years the oldest patient. A mild male preponderance was observed with male to female ratio of $1.66: 1$.

Despite the youngest patient in this study was observed in signet ring adenocarcinoma (poorly differentiated; 15 years), the younger age group patients were suffering from well differentiated adenocarcinoma (mean age: 43.2 years) followed by poorly differentiated adenocarcinoma (mean age: 46.6 years) and moderately differentiated adenocarcinoma (mean age: 54.15 years). Similarly, the mean age for diffuse type of adenocarcinoma was 38.0 years where as it was 49.5 years and 54.1 years for intestinal type

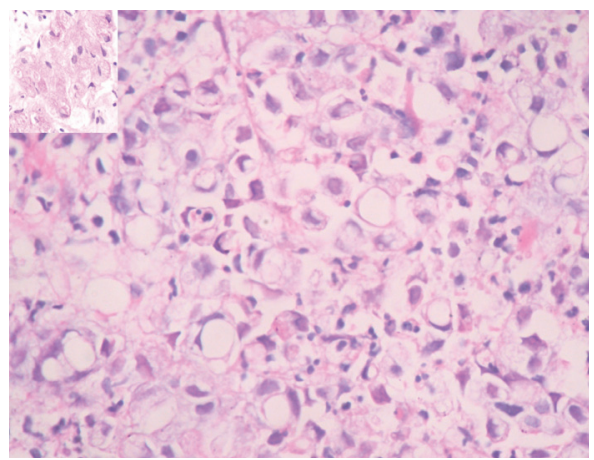

Figure 1: Photomicrograph showing sheets of signet ring cells with clear vacuolated cytoplasm and eccentrically placed nucleus (HE Stain, X400). Inset showing PAS positive signet ring cells.

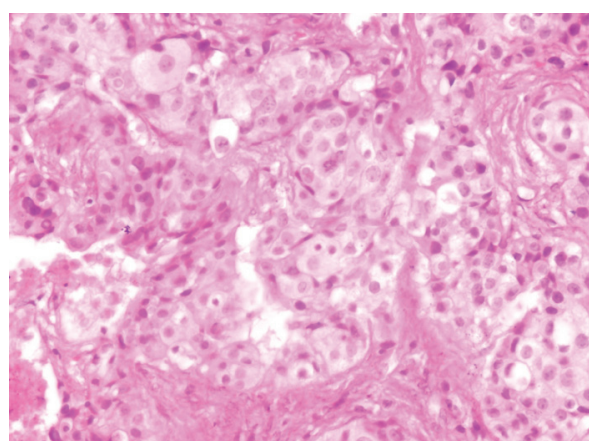

Figure 2: Microscopic picture of Hepatoid adenocarcinoma showing polygonal tumor cells with large central nuclei, prominent nucleoli, and abundant eosinophilic cytoplasm (HE Stain, X400).

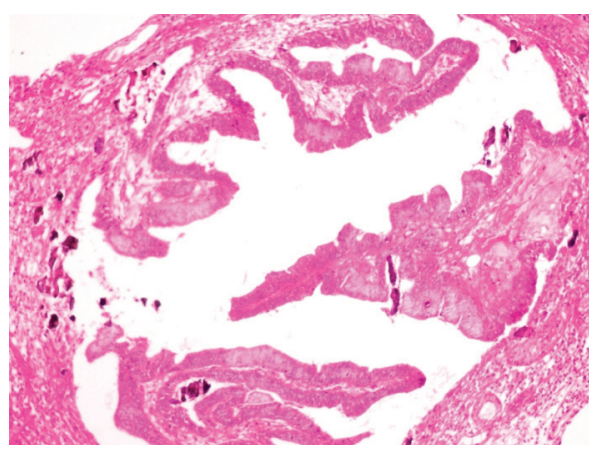

Figure 3: Microscopic picture of Papillary adenocarcinoma showing tumor cells arranged in Papillary pattern with fibrovascular core (HE Stain, X100). Note several psamnoma bodies within and around the fibrovascular core.

and mixed type of adenocarcinoma respectively. However, male predominance was not observed in younger patients. The male to female ratio among the patients below 30 years of age was $1: 1$.

According to Lauren classification, 30 cases (61.2\%) were of intestinal type, 11 cases $(22.4 \%)$ were of diffuse type (fig. 1 ) and 8 cases $(16.3 \%)$ were mixed type of adenocarcinoma. According to WHO classification the most common subtype 
was tubular adenocarcinoma $(n=35 ; 71.5 \%)$ followed by signet ring type $(\mathrm{n}=11 ; 22.4 \%)$, and hepatoid (fig.2), papillary (fig.3) and mucinous subtypes $(\mathrm{n}=1 ; 2.04 \%)$. Depending on differentiation, most common cases ( 20 cases; $40.8 \%$ ) were moderately differentiated (fig. 4 ) followed by 19 cases $(38.7 \%)$ of poorly differentiated adenocarcinoma and 10 cases (20.5\%) of well differentiated adenocarcinoma (fig.5). Among all diagnosed case of adenocarcinoma 2 cases $(4.08 \%)$ were early adenocarcinoma (fig. 6 ) whereas $47(97.92 \%)$ cases were advanced adenocarcinoma.

\section{DISCUSSION}

Stomach cancer is $2^{\text {nd }}$ most common cancer in males and $3^{\text {rd }}$ most cancer in females in Asia and worldwide. ${ }^{2}$ Carcinoma stomach is still the one of the most common cause of cancer related death in Nepal. It is the third most common cancer in males and also the one of the leading cause of death in females., ${ }^{2,3}$ In Nepal every year 27.8 new cases of cancers are diagnosed per 1000 population. ${ }^{2}$ In this study, 14.4 out of 1000 patients undergoing upper gastroendoscopy had gastric cancer. The mean age of the patients with adenocarcinoma was 47.6 years with a male preponderance. Ten patients (20.5\%) were below 40 years of age. A study done by Shrestha D et al. ${ }^{11}$ found $92.9 \%$ patients were above 40 years of age with male to female ratio $2: 1$. Similar finding was observed in other study which showed $19.5 \%$ patients of less than 45 years and male to female ratio was 1.97:1.12 Male predominance was lost in patients younger than 30 years of age. Similar finding was observed by a study done by $\mathrm{Wu} \mathrm{H}$ et al. ${ }^{13}$

According to the classification proposed by Lauren in 1965, gastric cancer has two histologic subtypes: Intestinal and Diffuse. ${ }^{14}$ The most common type of gastric cancer in this study was intestinal type followed by mixed type and diffuse type. $\mathrm{Wu} \mathrm{H}$ et al. in his study found $74 \%$ to be intestinal type and $16 \%$ diffuse type which concord with this study. ${ }^{13}$ In this study diffuse type adenocarcinoma was seen in younger patients in comparison with intestinal-type cancer. Lauren himself observed a stronger male preponderance and older age at diagnosis for intestinal-typecancer than for diffusetype cancer ${ }^{14}$, which was corroborated by HanaiA et al. ${ }^{15}$

Prognostic factors on survival includes tumor location, depth of penetration, lymph node status, Lauren classification, histological grade, micro vessel invasion, type ofresection performed, and use of postoperative chemotherapy and radiation therapy. ${ }^{16}$ In this study $40.8 \%$ cases were moderately differentiated, $38.7 \%$ cases were poorly differentiated adenocarcinoma and $20.5 \%$ cases were well differentiated adenocarcinoma. Pesic $\mathrm{M}$ et al in his study found $35.23 \%$ poorly differentiated, $34.29 \%$ moderately differentiated and $30.48 \%$ well differentiated adenocarcinoma. ${ }^{17}$

The dominant histologic type of cancer was adenocarcinoma

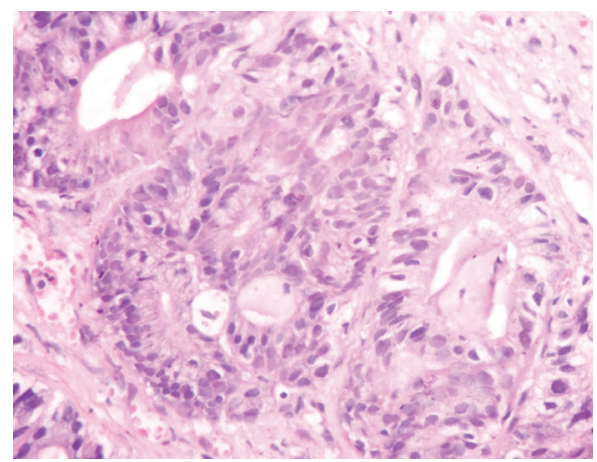

Figure 4: Photomicrograph showing tumor cells arranged in well-defined and poorly formed tubular pattern (HE Stain, X400).

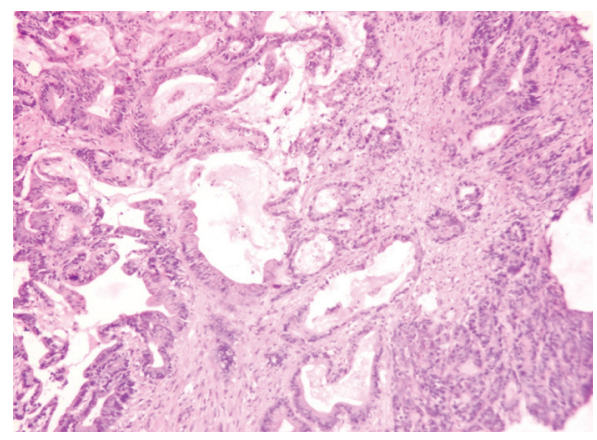

Figure 5: Photomicrograph showing tumor cells arranged in well-formed tubular pattern with intraluminal secretions. (HE Stain, X100).

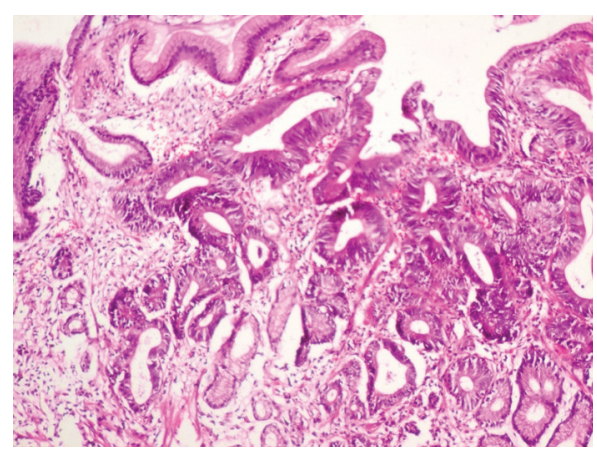

Figure 6: Photomicrograph showing early gastric carcinoma involving the mucosa and lamina propria only (HE Stain, X100).

in which tubular subtype predominated $(71.5 \%)$. Similar finding was observed by study done by Pallil D et al. ${ }^{18}$ who found $74 \%$ of cancer being adenocarcinoma. Signet ringtype was the second most frequent type of adenocarcinoma which was PAS stain positive (fig.2). It was observed in the early age group patients. Similar observation was documented by other studies. ${ }^{13-15}$

Hepatoid adenocarcinoma (HAC) is a special type of extrahepatic adenocarcinoma, which has a striking morphologic similarity to hepatocellular carcinoma. Since itsfirst description in the stomach, which is the most common location, it has been described in different organs such as lung, pancreas, esophagus, papilla of Vater, colon, urinary 
bladder, renal pelvis, ovaries, uterus, and cervix. ${ }^{19}$ In this study among all 49 cases of adenocarcinoma one case was of hepatoid adenocarcinoma. HAC consisted of polygonal tumor cells with large central nuclei, prominent nucleoli, and abundant eosinophilic cytoplasm (fig. 2). Serum alphafetoprotein(AFP) was elevated $(1550 \mathrm{IU} / \mathrm{mL})$ in this patient. CT scan revealed involvement of gastric lymph nodes but without involvement of liver. Most patients with HAC show an elevated AFP serum level, but normal serum levels have also been reported. ${ }^{20}$

Mucinous adenocarcinomas (MAs) of various origins often have a similar histologic appearance. They are composed of glands lined with either columnar mucin producing cells with abundant extracellular mucin accumulation $(>50 \%$ of tumor mass) or mucin-containing signet ring cells. ${ }^{21}$ In our study 1 case was of MA which comprises of sheets of signet ring cells containing mucin and also pool of extracellular mucin. Since the diagnosis was based on endoscopic biopsy specimen, which doesn't represent most of the tumor mass, the diagnosis of adenocarcinoma with lesser area showing mucin could be the another possibility.

Papillary gastric carcinoma (PGC) is a rare histologic entity among gastric adenocarcinomas. ${ }^{22}$ Although papillary in the thyroid or ovary occasionally exhibit psammoma bodies, papillary carcinoma of stomach is not known to demonstrate such psammoma bodies. ${ }^{23}$ In this study one case of papillary adenocarcinoma was found. Microscopically the tumor was arranged in papillary pattern with fibrovascular core. Calcific bodies were found in the surrounding fibrocollagenous tissue and fibrovascular core as concentrically laminated psammoma bodies which were seen singly or in clusters (fig. 3).

Among 49 cases of diagnosed gastric adenocarcinoma, one case was diagnosed as early gastric adenocarcinoma (fig.6). Early gastric carcinoma is defined as invasive carcinoma confined to mucosa and/or submucosa, with or without lymph node metastases, irrespective of the tumor size. ${ }^{24}$ Histologically, the most common forms of early gastric carcinoma are well differentiated, mostly with tubular and papillary architecture. The distinction between well-differentiated carcinoma and high grade dysplasia or carcinoma in situ can be challenging when only mucosal tissue is available for histologic assessment. Intramucosal invasion may not be as easily confirmed as an invasive carcinoma into submucosa where stromal desmoplasia is usually evident. The distinction between intramucosal carcinoma and carcinoma in situ or high grade dysplasia is important, as the intramucosal carcinoma of stomach, unlike the intramucosal carcinoma in the colon, does metastasize. ${ }^{25}$

H. Pylori have been classified as definite carcinogen by WHO. It has been shown to induce changes in the gastric mucosa and the gastric flora predisposing to the development of carcinoma. ${ }^{26}$ In this study among 49 cases of gastric cancer, 39 cases $(79.5 \%)$ were H. Pylori positive in Giemsa stain. This contrast with the findings done by Uemura Net al. ${ }^{8}$ They studied 1526 Japanese patients who had duodenal ulcers, gastric ulcers, gastric hyperplasia, or non ulcer dyspepsia, out of which 1246 had H. pylori infection and 280 did not. All the patients were followedup. Gastric cancers developed in 36 patients of the infected and none of the uninfected patients. ${ }^{8}$ Similar findings were observed by Enomoto et al. ${ }^{27}$ who found 98\% patients with gastric cancer were infected by H. Pylori. In Africa and South Asia, however, the incidence of gastric cancer in these areas is much lower than in other countries in spite of the high prevalence of the H. pylori infection. ${ }^{28} \mathrm{H}$. pylori have experienced relatively separate evolution processes, resulting in genomic diversity and differential potential for carcinogenesis. ${ }^{29}$ Furthermore, H. Pylori is not the sole cause of gastric cancer. Other factors associated with an increased risk of gastric cancer include chronic atrophic gastritis, hypertrophic gastropathy, gastric polyps, low socioeconomic status, and obesity. ${ }^{26,30}$

\section{CONCLUSION}

This study shows that Gastric carcinoma is a male predominant neoplasm usually of old age but can occur at younger ages. It predominantly occurs in H. Pylori infected patients and $\mathrm{H}$. Pylori eradication will help to decrease the incidence rate and mortality of stomach cancer.

\section{REFERENCES}

1. Parkin DM. International variation. Oncogene 2004;23:6329-40.

2. Ferlay J, Shin HR, Bray F et al. Estimates of worldwide burden of cancer in 2008: GLOBOCAN 2008. Int J Cancer 2010;127:2893917.

3. KK, Baral M, Shrestha BM. Multi-institution Hospital-based Cancer Incidence Data forNepal - An Initial Report.Asian Pacific J Cancer Pre 2010;10:259-62.

4. Jemal A, Bray F, Melissa M, Ferlay J, Ward E, Forman D. Global cancer statistics. Cancer j clin 2011;61:69-90.

5. IARC. Infection with Helicobacter pylori. In: IARC monographs on the evaluation of the carcinogenic risks to humans. Vol. 61. IARC; Lyon, France:1994. pp177-241.

6. Huang J-Q, Sridhar S, Chen Y, Hunt RH. Meta-analysis of the relationship between Helicobacter pylori seropositivity and gastric cancer. Gastroenterology 1999;114:1169-79.

7. Danesh J. Helicobacter pylori infection and gastric cancer: systematic review of the epidemiological studies. Aliment PharmacolTher 1999;13:851-6.

8. UemuraN, Okamoto S, Yamamoto $\mathrm{S}$ et al. Helicobacter Pylori Infection And The Development Of Gastric Cancer. N Engl J Med 2001;345:784-9.

9. IARC. Schistosomes, liver flukes and Helicobacter pylori. In: IARC Monographs on the Evaluation of Carcinogenic Risks to Humans Vol. 61. IARC; Lyon, France:1994. pp61.

10. Bing H, Nassim EH, Scott S, Nancy L, Robert B, Aurelia ME. Gastric cancer: Classification, histology and application of molecular 
pathology. J GastrointestOncol 2012;3:251-61.

11. Shrestha D, Shrestha R, Shrestha S, CSK.Gastric cancer in a tertiary referral center: Ten years' experience. Post-Graduate Medical Journal of NAMS2009 (Cited 11 November 2012). Available from: http:// www.pmjn.org.np/index.php/pmjn/article/view/69\#

12. Khan D, Hassan MK, Rehman A, Khan A, Altaf M, Alam F. Gastric carcinoma: location, morphological and histological profile. J postgrad med inst 2012;26:170-5.

13. Wu H, Rusiecki JA, Zhu K,Potter J, Devesa SS. Stomach Carcinoma Incidence Patterns in the United States by Histologic Type and Anatomic Site. Cancer Epidemiol Biomarkers Prev 2009;18:194552 .

14. Lauren P. The two histological main types of gastric carcinoma: diffuse and so-called intestinal-type carcinoma. Acta Pathol Microbiol Scand 1965;64:31-49.

15. Hanai A, Fujimoto I, Taniguchi H. Trends of stomach cancer incidence and histologic types in Osaka. In: Trends in Cancer Incidence.Causes and Practical Implications. K. Magnus (ed.). Hemisphere Publishing Corp., Washington DC: 1982. pp143-54.

16. Schlemper RJ, Itabashi M, Kato Y. Differences and diagnostic criteria for gastric carcinoma between Japanese and western pathologists. Lancet 1997;349:1725-9.

17. Pesic M, Karanikolic A, Dorevic N et al. The importance of primary gastric cancer location in 5-year survival rate. Arch Oncol 2001;12:51-3.

18. Pallil D,Bianchi S, Cipriani $\mathrm{F}$ et al. Reproducibility of histologic classification of gastric cancer. Br J Cancer 1991;63:765-8.

19. Terracciano LM, Glatz K, Mhawech P et al.Hepatoid Adenocarcinoma With Liver Metastasis Mimicking Hepatocellular Carcinoma An Immunohistochemical and Molecular Study of Eight Cases. Am J Surg Pathol 2003:27;1302-12.
20. Ishikura H, Kishimoto T, Andachi H et al. Gastrointestinal hepatoid adenocarcinoma: venous permeation and mimicry of hepatocellular carcinoma, a report of four cases. Histopathology 1997;31:47-54.

21. Peiguo GC, Lawrence C, Lawrence MW, Sean KL. Determining the Site of Origin of Mucinous Adenocarcinoma: An ImmunohistochemicalStudy of 175 Cases. Am J Surg Pathol 2011;35:1830-6.

22. Yasuda K, Adachi Y, Shiraishi N, Maeo S, Kitano S. Papillary adenocarcinoma of the stomach. Gastric Cancer 2000;3:33-8.

23. Park SY, Ko GH, Kim WH, Kim YII. Papillary adenocarcinoma of stomach with psammoma bodies: Report of two cases. J Korean Med Sci 1999;14:213-6.

24. Hamilton R, Aatonen LA. Tumors of Digestive System. Lyon:IARC; 2000:39-52.

25. Hu B, Hajj NE, Sittler S, Lammert N, Barnes R,Meloni-Ehrig A. Gastric cancer: Classification, histology and application of molecular pathology. J Gastrointest Oncol 2012;3:251-61.

26. Fenogilo-Preiser C, Carneiro F, Correa $\mathrm{P}$ et al. Gastric carcinoma. In: Hamilton S, Aaltonin L, eds. Pathology and Genetics. Tumors of the Digestive System, vol 1.Lyon Press; Lyon, France:2000.pp37-52.

27. Enomoto H, Watanabe H, Nishikura K, Umezawa H, Asakura H. Topographic distribution of Helicobacter pylori in the resected stomach. Eur J GastroenterolHepatol 1998;10:473-8.

28. Alm RA, Ling LS, Moir DT et al. Genomic-sequence comparison of two unrelated isolates of the human gastric pathogen Helicobacter pylori. Nature 1999;397:176-80.

29. Dong QJ, Zhan SH, Wang LL, Xin YN, Jiang M, Xuan SY. Relatedness of Helicobacter pylori populations to gastric carcinogenesis. World J Gastroenterol 2012;18:6571-6.

30. Gore R. Gastrointestinal cancer. RadiolClin North Am 1997;35:295310. 